\title{
Fatores Determinantes de Morbilidade nos Doentes com Lúpus Eritematoso Sistémico
}

\author{
Determinant Factors of Morbidity in Patients with \\ Systemic Lupus Erythematosus
}

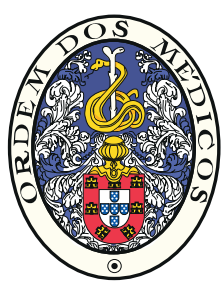

\author{
Margarida JACINTO $\square^{1,2,3}$, Eliana SILVA ${ }^{1,4}$, Nuno RISO ${ }^{1}$, Maria Francisca MORAES-FONTES ${ }^{1}$ \\ Acta Med Port 2017 May;30(5):368-372 - https://doi.org/10.20344/amp.8082
}

\section{RESUMO}

Introdução: O lúpus eritematoso sistémico pode apresentar uma gravidade variável. Contudo, não existem biomarcadores que preveem o curso da doença. O dano é medido pelo índice Systemic Lupus International Collaborating Clinics/Systemic Damage Index que define a gravidade e prevê o seu prognóstico.

Objetivo: Avaliação dos fatores que determinam dano nos doentes com lúpus eritematoso sistémico.

Material e Métodos: Estudo retrospetivo, monocêntrico, em doentes com lúpus eritematoso sistémico ( $\geq 4$ critérios do American College of Rheumatology $-100 \%$ dos doentes, $n=76$ ), do sexo feminino, seguidos por um período $\geq 5$ anos. Início da doença, etnia, duração, número de critérios American College of Rheumatology no final do seguimento, fenótipo renal, neuropsiquiátrico (e articular, co-morbilidades e Systemic Lupus Erythematosus Disease Activity Index -2K foram correlacionados com a presença e grau de dano medido pelo índice Systemic Lupus International Collaborating Clinics/Systemic Damage Index. A acumulação de critérios American College of Rheumatology foi objetivada num sub-grupo de doentes seguidos desde o início. A análise estatística utilizou o qui-quadrado, Wilcoxon Mann-Whitney e a correlação de Spearman $(p<0,05)$.

Resultados: O Systemic Lupus International Collaborating Clinics Index era superior a 0 em $56,5 \%$ dos doentes. Estes doentes tinham um maior tempo de doença, um maior número de critérios American College of Rheumatology e um fenótipo neuropsiquiátrico, quando comparados com doentes sem dano $(p<0,05)$. Verificou-se uma correlação positiva entre o valor numérico de critérios American College of Rheumatology acumulados no final do seguimento e a atividade da doença nos últimos cinco anos (Spearman rho 0,02 e $p<0,05)$. Não se verificaram diferenças em relação às outras variáveis.

Discussão e Conclusão: A duração da doença e o número de critérios do American College of Rheumatology acumulados conseguem prever a presença de dano. A doença neuropsiquiátrica teve impacto na morbilidade dos doentes com lúpus eritematoso sistémico, identificando um subgrupo em risco.

Palavras-chave: Actividades da Vida Diária; Indicadores de Saúde; Lupus Eritematoso Sistémico; Morbilidade; Portugal; Qualidade de Vida

\section{ABSTRACT}

Introduction: Severity in systemic lupus erythematosus may vary from mild to even fatal consequences. There are no biomarkers to predict the disease's prognosis. The Systemic Lupus International Collaborating Clinics/ Systemic Damage Index defines systemic lupus erythematosus disease severity and is found to predict prognosis.

Objective: To test damage determinants in a single-centre systemic lupus erythematosus cohort.

Material and Methods: Retrospectively followed systemic lupus erythematosus female patients (defined by the identification of at least four systemic lupus erythematosus American College of Rheumatology criteria - fulfillment $100 \%, n=76$ ) over the past five years. Age of onset, ethnicity, disease duration, number of American College of Rheumatology criteria at the end of follow-up, cumulative: renal, neuropsychiatric and articular phenotypes, hypertension, dyslipidaemia, smoking and Systemic Lupus Erythematosus Disease Activity Index $2 \mathrm{~K}$ were correlated to the presence and degree of irreversible damage (Systemic Lupus International Collaborating Clinics Damage Index). Accumulation of American College of Rheumatology criteria was measured in a sub-group of patients followed from disease onset (within a year of the first symptom ascribed to systemic lupus erythematosus) ( $n=39-51 \%$ ); Systemic Lupus Erythematosus Disease Activity Index and Systemic Lupus International Collaborating Clinics Damage Index were performed. Statistical analysis was performed using Chi-square, Wilcoxon Mann-Whitney tests and Spearman correlation rho (Sig. 2-tailed $p<0.05$ ).

Results: Systemic Lupus International Collaborating Clinics/Systemic Damage Index $>0$ was present in $56.6 \%$ and significantly associated to a longer duration, a higher number of American College of Rheumatology criteria and a neuropsychiatric phenotype when compared with those with no damage. The final number of American College of Rheumatology criteria accrued was positively correlated to a higher disease activity over the past five years of follow-up (Spearman's rho 0.02 and $p<0.05$ ). There was no effect from other features.

Discussion and Conclusion: Disease duration and number of American College of Rheumatology criteria predict Systemic Lupus International Collaborating Clinics/ Systemic Damage Index. neuropsychiatric disease has an impact on damage accrual.

Keywords: Activities of Daily Living; Health Status Indicators; Lupus Erythematosus, Systemic; Morbidity; Portugal; Quality of Life

\footnotetext{
1. Unidade de Doenças Auto-imunes. Serviço Medicina 7.2. Hospital Curry Cabral. Centro Hospitalar de Lisboa Central. Lisboa. Portugal.

2. Serviço de Medicina Interna. Hospital Espírito Santo. Évora. Portugal.

3. Núcleo de Estudos de Doenças Auto-imunes. Sociedade Portuguesa de Medicina Interna. Lisboa. Portugal.

4. Serviço de Patologia Clínica. Instituto Português de Oncologia Francisco Gentil. Lisboa. Portugal.

$\triangle$ Autor correspondente: Margarida Jacinto. margarida.jacinto14@gmail.com

Recebido: 28 de julho de 2016 - Aceite: 22 de novembro de 2016 | Copyright @ Ordem dos Médicos 2017
} 


\section{INTRODUÇÃO}

O lúpus eritematoso sistémico (LES) é uma doença autoimune heterogénea, multi-sistémica, que engloba a quase totalidade dos aparelhos e sistemas, com gravidade variável, de ligeira a fatal. Estima-se que a prevalência em Portugal seja de 100 a 200/100 000 habitantes, ${ }^{1}$ superior aos restantes países na Europa do Sul onde varia entre 34 - 91 casos/100 000 habitantes. $^{2}$ O LES afeta predominantemente o sexo feminino num rácio de cerca de 9:1, e em mais de $80 \%$, ocorre em mulheres em idade fértil. A história natural da doença caracteriza-se por um curso ondulante de episódios de recidivas intercalados com períodos de remissão, com um prognóstico variável ${ }^{3}$ e que ocorrem mesmo sob terapêutica. Nas últimas décadas, tem-se verificado uma redução da mortalidade devido à doença e está descrita uma remissão progressiva a longo prazo, sendo os eventos trombóticos a causa de morte mais frequente. ${ }^{4}$ Desta forma, a presença de co-morbilidades cardiovasculares (hipertensão arterial, tabagismo e dislipidemia) ocupa um papel de relevo na avaliação de doentes com LES.

O LES pode ser avaliado em duas dimensões: através da atividade da doença e da lesão órgão-alvo acumulada. Para a primeira deve ser utilizado um instrumento estandardizado, válido, fiável, sensível à mudança e viável na prática clínica corrente, de forma a ser aplicado na consulta médica. Apesar de existirem vários índices de atividade validados, o Systemic Lupus Erythematosus Disease Activity Index (SLEDAl), ${ }^{5}$ que tem vindo a ser ajustado (SLEDAl $2 \mathrm{~K})^{6}$ constitui o instrumento clínico mais facilmente utilizado, válido mesmo quando calculado retrospetivamente. ${ }^{7}$ É um índice quantitativo cuja pontuação varia entre 0 e 105, que avalia a presença de manifestações clínicas e laboratoriais de atividade lúpica, presentes à data da avaliação ou nos 10 dias precedentes. Considera-se a doença ativa quando o índice é igual ou superior a três. ${ }^{8}$ Para a segunda, na avaliação do dano (determinado pela presença de lesões irreversíveis), o instrumento validado é o Systemic Lupus International Collaborating Clinics/American College of Rheumatology Damage Index (SLICC/ACR) ${ }^{9}$ que quantifica as lesões acumuladas após o diagnóstico de LES, presentes por mais de seis meses, não pretendendo estabelecer uma relação causal com a doença. O seu valor pode variar entre 0 e 49. São avaliados 12 domínios, sendo atribuída a pontuação 1 se houver lesão irreversível. No caso de eventos repetidos, com um intervalo de tempo superior a seis meses [por ex. acidente vascular cerebral (AVC), enfarte agudo do miocárdio (EAM), necrose avascular, neoplasia], é atribuída pontuação a cada evento. O valor máximo no domínio renal é três, no caso de insuficiência renal terminal. Ao longo do tempo, o valor do SLICC pode aumentar mas por definição nunca diminui. Isto significa que, mesmo que sejam efetuadas medidas corretivas (por ex. cirurgia de catarata ou artroplastia da articulação afetada por necrose avascular), o dano continua a ser contabilizado. É recomendada uma avaliação basal e, de seguida, com uma frequência anual. À semelhança do SLEDAI, mantém a sua validade quando documentado de forma retrospetiva. ${ }^{10}$
No LES não existem biomarcadores que possam prever a progressão da doença. Na prática clínica, a lesão de órgão-alvo acumulada, ou seja, o índice SLICC/Systemic Damage Index (SDI), tem sido a única medida usada para definir a gravidade da doença pois está comprovadamente associado a acréscimo de dano futuro e mortalidade..$^{11-15}$ Vários estudos identificaram fatores de mau prognóstico na doença, sendo globalmente reconhecidos: a idade jovem à data do diagnóstico, o género, o estatuto socioeconómi$\mathrm{Co}$, a etnia e a atividade da doença medida pelo SLEDAI. A idade de início correlaciona-se com a expressão clínica, o padrão de órgãos atingidos e os achados serológicos. Alguns estudos tentaram definir quais os determinantes da morbilidade e mortalidade nos doentes com LES. Foram destacados: atividade da doença (artrite, eritema malar, nefropatia ativa, febre, envolvimento neurológico, fenómeno Raynaud, serosite, trombocitopenia e trombose), infeções, hipertensão arterial, dislipidemia, tabagismo, osteoporose, citopenias relacionadas com o uso de imunossupressores e neoplasias. ${ }^{16-18}$

Para efeitos de estudos observacionais são habitualmente incluídos doentes com um número mínimo de quatro critérios ACR de classificação de LES, ${ }^{19}$ preenchidos de forma cumulativa, ao longo do seguimento clínico, sem uma duração de acumulação de critérios pré-definida. Até à presente data, não foi desenvolvido um índice que permita relacionar a forma global de apresentação da doença com o seu prognóstico. Neste estudo de doentes com LES foi efetuada uma caracterização demográfica e clínica juntamente com fatores que potencialmente determinam dano, mas não fazem parte do SLICC/SDI, tais como a etnia, o número de critérios $\mathrm{ACR}$ que os doentes acumularam durante os primeiros seis meses da doença, a presença de hipertensão arterial, dislipidemia e tabagismo.

\section{MATERIAL E MÉTODOS}

Trata-se de um estudo retrospetivo de doentes de género feminino seguidos na Consulta Externa da Unidade de Doenças Auto-imunes do Hospital Curry Cabral, Centro Hospitalar Lisboa Central, de 1 de janeiro de 2009 a 31 de dezembro de 2014, com pelo menos uma consulta no ano de 2014. Todos apresentavam o diagnóstico de LES, segundo os critérios de classificação do American College of Rheumatology (ACR) 1997, cumprindo pelo menos quatro dos 11 critérios ( $n=76$ doentes, 100\%). As variáveis consideradas foram: a idade de início da doença, a etnia, a duração da doença, o número de critérios $A C R$ acumulados nos primeiros seis meses de doença e no final do follow-up, a hipertensão, a dislipidemia e o tabagismo. Os doentes classificados de acordo com o principal fenótipo clínico: neuropsiquiátrico (NP), renal ou articular. ${ }^{20,21}$

Com uma frequência semestral, foi retrospetivamente efetuado o cálculo da atividade da doença, utilizando uma modificação do índice SLEDAI original, o SLEDAI 2K. O SLICC/ACR foi o instrumento utilizado para quantificar as lesões acumuladas após o diagnóstico de LES, calculado 
Tabela 1 - Características clínicas dos doentes com lúpus eritematoso sistémico

\begin{tabular}{|c|c|}
\hline Características demográficas e clínicas & \\
\hline Idade no início da doença (média \pm desvio padrão) & $32 \pm 12$ \\
\hline Não-caucasiano (n, \%) & $9(11,8)$ \\
\hline Duração da doença (anos) (média \pm desvio padrão) & $17 \pm 7,9$ \\
\hline Hipertensão (n, \%) & $44(57,9)$ \\
\hline Dislipidemia (n, \%) & $35(46,1)$ \\
\hline Hábitos tabágicos (n, \%) & $16(21,1)$ \\
\hline Neoplasia (n, \%) & $5(6,6)$ \\
\hline Infeção (n, \%) & $41(53,9)$ \\
\hline Alterações neuropsiquiátricas (n, \%) & $16(21,1)$ \\
\hline Alteração renal (n, \%) & $31(40,8)$ \\
\hline Alterações articulares (n, \%) & $56(73,7)$ \\
\hline ANA $(n, \%)$ & $76(100)$ \\
\hline Anti-dsDNA (n, \%) & $58(76,3)$ \\
\hline $\begin{array}{l}\text { Média do SLEDAI } \\
\text { (avaliação semestral, durante } 5 \text { anos) }\end{array}$ & $3,1 \pm 0,5$ \\
\hline Critérios ACR acumulados - final & $5,4 \pm 1,3$ \\
\hline
\end{tabular}

anualmente, na nossa população. Os doentes foram divididos de acordo com a presença de dano (SLICC $=0$, sem dano; SLICC > 0, com dano) sendo realizada a correlação com as variáveis estudadas em cada um dos grupos.

Todos os dados foram obtidos através da consulta dos processos clínicos em formato papel e pelo programa informático - SCLINICO. Foi utilizado o Statistical Product and Service Solutions (SPSS) para a análise estatística: teste de qui-quadrado e de Wilcoxon-Mann-Whitney (dados de distribuição não paramétrica) e a correlação de Spearman rho para o teste de associação entre variáveis lineares.
Tabela 2 - Avaliação retrospetiva do SLEDAI (valor semestral)

\begin{tabular}{ccc}
\hline Ano de avaliação & $\begin{array}{c}\text { SLEDAI } \\
\mathbf{1}^{\circ} \text { semestre }\end{array}$ & $\begin{array}{c}\text { SLEDAI } \\
\mathbf{2}^{\circ} \text { semestre }\end{array}$ \\
\hline 2010 & 4,36 & 3,24 \\
2011 & 3,48 & 3,49 \\
2012 & 3,02 & 2,28 \\
2013 & 2,54 & 2,71 \\
\hline
\end{tabular}

\section{RESULTADOS}

Durante a realização do estudo foram seguidos cento e um doentes com o diagnóstico de LES segundo os critérios ACR na nossa unidade. Noventa e três eram do sexo feminino e 84 eram seguidas, pelo menos desde 2009, na consulta externa. A nossa amostra de 76 doentes cumpriu estas quatro premissas: diagnóstico de LES segundo critérios ACR, género feminino, diagnóstico pelo menos desde 2009 e pelo menos uma consulta no ano de 2014. Os dados demográficos e clínicos são apresentados na Tabela 1. Na altura do diagnóstico de LES, a média de idade dos doentes era de 32 anos, com um desvio padrão de 12, sendo a idade mínima de 11 e a máxima de 66 . À data da realização do estudo, a idade média dos doentes era de 49 anos, com um desvio padrão de 13 , sendo a idade mínima de 23 anos, a idade máxima de 79 anos. Em média, os doentes tinham 17 anos de duração da doença (desvio padrão de 7,92 anos, mínimo de cinco anos e máximo de 36 anos), sendo seguidos na unidade em média há cerca de 12,62 anos (desvio padrão de 4,86, mínimo cinco anos e máximo de 22 anos). Cerca de um terço das doentes era solteira (28 doentes - 36,84\%). Nove doentes $(11,8 \%)$ eram de etnia não-caucasiana, predominantemente de descendência africana. No final do seguimento, a média do ACR acumulado era de 5,37 (desvio padrão de 1,32, com um

Tabela 3 - Comparação entre os doentes com e sem dano (de acordo com o índice SLICC/SDI)

\begin{tabular}{|c|c|c|c|c|}
\hline Características & $\begin{array}{l}\text { SLICC }=0 \\
(n=43)\end{array}$ & $\begin{array}{l}\text { SLICC } \geq 1 \\
(n=33)\end{array}$ & $p\left(\right.$ Sig $\left.^{*}\right)$ & Teste estatístico \\
\hline Idade no início da doença (média \pm desvio padrão) & $32 \pm 11$ & $31 \pm 13$ & 0,653 & MW \\
\hline Não-caucasiano (n, \%) & $4(44,4)$ & $5(55,6)$ & 0,610 & PCS \\
\hline Duração da doença (anos) (média \pm desvio padrão) & $14 \pm 7$ & $19 \pm 8$ & $0,007^{*}$ & MW \\
\hline Hipertensão (n, \%) & $16(36,4)$ & $27(61,4)$ & 0,338 & PCS \\
\hline Dislipidemia (n, \%) & $12(34,3)$ & $22(62,9)$ & 0,201 & PCS \\
\hline Hábitos tabágicos (n, \%) & $7(43,8)$ & $9(56,2)$ & 1,000 & PCS \\
\hline Neoplasia (n, \%) & $0(0)$ & $6(100)$ & 0,111 & PCS \\
\hline Infeção (n, \%) & $16(39)$ & $24(58,5)$ & 0,809 & PCS \\
\hline Alterações neuropsiquiátricas (n, \%) & $2(11,1)$ & $16(88,9)$ & $0,004^{*}$ & PCS \\
\hline Alteração renal (n, \%) & $13(41,9)$ & $17(54,8)$ & 0,614 & PCS \\
\hline Alterações articulares (n, \%) & $22(39,3)$ & $33(58,9)$ & 0,631 & PCS \\
\hline ANA $(n, \%)$ & $32(42,1)$ & $43(56,6)$ & 1,000 & PCS \\
\hline Anti-dsDNA (n, \%) & $24(41,4)$ & $33(56,9)$ & 1,000 & PCS \\
\hline Média do SLEDAI (avaliação semestral, durante 5 anos) & $2,2 \pm 2,0$ & $3,6 \pm 2,9$ & 0,054 & MW \\
\hline $\begin{array}{l}\text { Critérios ACR acumulativos - nos primeiros } 6 \text { meses } \\
\text { após o diagnóstico ( } \mathrm{n} \text { disponível) }\end{array}$ & $\begin{array}{l}4,1 \pm 1,3 \\
(n=21)\end{array}$ & $\begin{array}{l}3,7 \pm 1,5 \\
(\mathrm{n}=18)\end{array}$ & 0,280 & MW \\
\hline Critérios ACR acumulados - final & $5,0 \pm 1,1$ & $5,6 \pm 1,4$ & $0,046^{*}$ & MW \\
\hline
\end{tabular}

MW: Mann-Whitney; PCS: Pearson Chi-square 
mínimo de quatro e máximo de 10). Quarenta e três doentes $(56,58 \%)$ apresentavam dano (SLICC > 0). A média de atividade da doença (SLEDAI $2 \mathrm{~K} \geq 3$ ) ao longo dos últimos cinco anos (Tabela 2) não se correlacionou com o dano encontrado no final do seguimento clínico, nem mesmo quando a análise foi realizada de acordo com a duração da doença (17 doentes com LES há menos de 10 anos; 32 doentes com duração entre 10 e 19 anos e 27 doentes com LES há pelo menos 20 anos). A presença de dano estava associada de forma estatisticamente significativa a uma maior duração de doença, um maior número de critérios ACR e um fenótipo NP (Tabela 3) sem relação com as outras características demográficas e clínicas. O número final de critérios ACR acumulados mais elevado foi positivamente correlacionado com a atividade da doença nos últimos cinco anos de follow-up (Spearman rho 0,02).

\section{DISCUSSÃO E CONCLUSÃO}

O grupo total de doentes com LES seguido na nossa unidade foi objeto de publicação prévia ${ }^{22}$ e é muito semeIhante ao registo ibérico de doentes com LES. ${ }^{23} \mathrm{Em}$ comparação, a frequência de dano no nosso grupo de doentes é semelhante mas o valor de SLICC é mais elevado, ${ }^{24}$ predominantemente determinado por manifestações neurológicas.

Na nossa coorte, a média de idade na altura do diagnóstico da doença e a percentagem de indivíduos não-caucasianos foi semelhante em ambos os grupos, com e sem dano, ao contrário do que seria expectável, pois o diagnóstico em idades tardias e a raça não-caucasiana parece ter um efeito modificador no prognóstico da doença. ${ }^{14,25}$ O envolvimento renal prévio, não está associado a dano na nossa coorte, à semelhança do registo SLICC. ${ }^{14}$ Surpreendentemente, ao contrário do esperado, a presença de fatores de risco cardiovascular, isto é, dislipidemia, tabagismo e hipertensão arterial, assim como a presença de neoplasias e infeções, não estiveram associados a maior morbilidade. Não encontramos relação entre os critérios ACR acumulados durante os primeiros seis meses de doença, mas devemos salientar que apenas foi possível recuperar esses dados em cerca de metade dos doentes em cada grupo (com e sem dano). Neste estudo, a percentagem de doentes com dano foi significativamente mais elevada no grupo de doentes com maior duração de doença e um

\section{REFERÊNCIAS}

1. Sociedade Portuguesa de Reumatologia. Reuma Census 2011-2013. [consultado 2016 jan 26]. Disponível em: http://www.reumacensus.org/ pdf/newsletter_42.pdf.

2. Danchenko N, Satia JA, Anthony MS. Epidemiology of systemic lupus erythematosus: a comparison of worldwide disease burden. Lupus. 2006;15:308-18.

3. Jimenez S, Cervera R, Font J, Ingelmo M. The epidemiology of systemic lupus erythematosus. Clin Rev Allergy Immunol. 2003;25:3-12.

4. Cervera R, Khamashta MA, Hughes GR. The Euro-lupus project: epidemiology of systemic lupus erythematosus in Europe. Lupus. 2009;18:869-74.

5. Bombardier C, Gladman DD, Urowitz MB, Caron D, Chang $\mathrm{CH}$. Derivation of the SLEDAI. A disease activity index for lupus patients. The Committee on Prognosis Studies in SLE. Arthritis Rheum. 1992;35:630- maior número de critérios ACR acumulados estando nestes associado maior dano, à semelhança de estudos prévios. ${ }^{14}$

O nosso estudo apresenta limitações por ser um estudo observacional e retrospetivo, no qual excluímos oito doentes de sexo masculino. Trata-se de um estudo monocêntrico, sendo que um aumento do número de doentes poderia trazer poder estatístico à correlação entre a média de SLEDAI nos últimos cinco anos e a presença de dano, valor que esteve perto de atingir significância estatística.

Com este trabalho verificámos que os doentes com maior morbilidade, com dano (SLICC > 0) tinham uma maior duração da doença, um fenótipo neuropsiquiátrico e um maior número de critérios ACR acumulados no final do seguimento clínico. As estratégias terapêuticas dirigidas à redução das suas manifestações e ao controlo da atividade da doença irão de encontro à possibilidade de reduzir a morbilidade e mortalidade que ainda hoje são encontradas nesta doença.

\section{OBSERVAÇÕES}

Este trabalho foi apresentado sob a forma de poster na Reunião do American College of Rheumatology (ACR/ ARPH) de 2015, publicado a 29 Setembro de 2015 no livro de abstracts.

\section{PROTECÇÃO DE PESSOAS E ANIMAIS}

Os autores declaram que os procedimentos seguidos estavam de acordo com os regulamentos estabelecidos pelos responsáveis da Comissão de Investigação Clínica e Ética e de acordo com a Declaração de Helsínquia da Associação Médica Mundial.

\section{CONFIDENCIALIDADE DOS DADOS}

Os autores declaram ter seguido os protocolos do seu centro de trabalho acerca da publicação de dados.

\section{CONFLITOS DE INTERESSE}

Os autores declaram não terem qualquer conflito de interesse relativamente ao presente artigo.

\section{FONTES DE FINANCIAMENTO}

Os autores declaram não ter recebido subsídios ou bolsas para a elaboração do artigo.

40.

6. Gladman DD, Ibanez D, Urowitz MB. Systemic lupus erythematosus disease activity index 2000. J Rheumatol. 2002;29:288-91.

7. FitzGerald JD, Grossman JM. Validity and reliability of retrospective assessment of disease activity and flare in observational cohorts of lupus patients. Lupus. 1999;8:638-44.

8. Yee CS, Farewell VT, Isenberg DA, Griffiths B, Teh LS, Bruce IN, et al. The use of Systemic Lupus Erythematosus Disease Activity Index-2000 to define active disease and minimal clinically meaningful change based on data from a large cohort of systemic lupus erythematosus patients. Rheumatology. 2011;50:982-8.

9. Gladman D, Ginzler E, Goldsmith C, Fortin P, Liang M, Urowitz M, et al. The development and initial validation of the Systemic Lupus International Collaborating Clinics/American College of Rheumatology 
damage index for systemic lupus erythematosus. Arthritis Rheum. 1996;39:363-9.

10. Bernatsky S, Clarke A, Abrahamowicz M, Neville C, Karp I, Pineau CA. A comparison of prospective and retrospective evaluations of the Systemic Lupus International Collaborating Clinics/American College of Rheumatology Damage Index for systemic lupus erythematosus. J Rheumatol. 2005;32:820-3.

11. Rahman P, Gladman DD, Urowitz MB, Hallett D, Tam LS. Early damage as measured by the SLICC/ACR damage index is a predictor of mortality in systemic lupus erythematosus. Lupus. 2001;10:93-6.

12. Cardoso CR, Signorelli FV, Papi JA, Salles GF. Initial and accrued damage as predictors of mortality in Brazilian patients with systemic lupus erythematosus: a cohort study. Lupus. 2008;17:1042-8.

13. Alarcon GS, Roseman JM, McGwin G Jr, Uribe A, Bastian HM, Fessler $\mathrm{BJ}$, et al. Systemic lupus erythematosus in three ethnic groups. XX. Damage as a predictor of further damage. Rheumatology. 2004;43:2025.

14. Bruce IN, O'Keeffe AG, Farewell V, Hanly JG, Manzi S, Su L, et al. Factors associated with damage accrual in patients with systemic lupus erythematosus: results from the Systemic Lupus International Collaborating Clinics (SLICC) Inception Cohort. Ann Rheum Dis. 2015;74:1706-13.

15. Kasitanon N, Magder LS, Petri M. Predictors of survival in systemic lupus erythematosus. Medicine. 2006;85:147-56.

16. Cervera R, Khamashta MA, Font J, Sebastiani GD, Gil A, Lavilla P, et al. Morbidity and mortality in systemic lupus erythematosus during a 10year period: a comparison of early and late manifestations in a cohort of 1,000 patients. Medicine. 2003;82:299-308.

17. Montes RA, Mocarzel LO, Lanzieri PG, Lopes LM, Carvalho A,
Almeida JR. Smoking and its association with morbidity in systemic lupus erythematosus evaluated by the Systemic Lupus International Collaborating Clinics/American College of Rheumatology Damage Index: preliminary data and systematic review. Arthritis Rheumatol. 2016;68:441-8.

18. Yu HH, Chen PC, Yang YH, Wang LC, Lee JH, Lin YT, et al. Statin reduces mortality and morbidity in systemic lupus erythematosus patients with hyperlipidemia: a nationwide population-based cohort study. Atherosclerosis. 2015;243:11-8.

19. Hochberg MC. Updating the American College of Rheumatology revised criteria for the classification of systemic lupus erythematosus. Arthritis Rheum. 1997;40:1725.

20. The American College of Rheumatology nomenclature and case definitions for neuropsychiatric lupus syndromes. Arthritis Rheum. 1999;42:599-608.

21. Weening JJ, D'Agati VD, Schwartz MM, Seshan SV, Alpers CE, Appel $\mathrm{GB}$, et al: The classification of glomerulonephritis in systemic lupus erythematosus revisited. Kidney Int. 2004;65:521-30.

22. Moraes-Fontes MF, Lucio I, Santos C, Campos MM, Riso N, Vaz Riscado M. Neuropsychiatric features of a cohort of patients with systemic lupus erythematosus. ISRN Rheumatol. 2012;2012:989218.

23. Goncalves MJ, Sousa S, Ines LS, Duarte C, Borges J, Silva C, et al: Characterization of damage in Portuguese lupus patients: analysis of a national lupus registry. Lupus. 2015;24:256-62.

24. Moraes-Fontes MF, Riso N. Characterization of damage in Portuguese lupus patients. Lupus. 2015;24:778.

25. Cervera R, Doria A, Amoura Z, Khamashta M, Schneider M, Guillemin F, et al. Patterns of systemic lupus erythematosus expression in Europe. Autoimmun Rev. 2014;13:621-9. 